\title{
Functional Approach for Hamiltonian Circuit and Graph Isomorphism Problems
}

\author{
R. T. Faizullin, Omsk State Technical University, r.t.faizullin@mail.ru
}

The aim of this work is to establish relation between well-known basic problems of cryptanalysis [1],[2] as Hamiltonian Circuit and Subgraph Isomorphism problems and global optimization problem for classes of functionals constructed as sums of low dimensional polynomials. Let's consider for arbitrary graph wellknown Hamiltonian Circuit problem ( way via circle vortex by vortex where vortexes is not equal). We can numerate the graph vortexes via prime numbers $r_{j}$ where $r_{1}=3$ and $r_{j+1}=2 r_{j}+s_{j}$. We have the sum: $R=\sum_{j=1}^{n} r_{j}$ from which we can recognize $r_{i}$ without order. Let's $\Upsilon_{i}$ is a set of contacted vortexes with vortex $i$ where $i$ is one of the numbers $r_{j}$.

Then we have next result:

Theorem 0.1 If there are $s$ Hamiltonian circuits with path numbers $t_{1}^{r}, . ., t_{n}^{r}, 1 \leq r \leq s$ then for every $m \geq 2$ from natural numbers global minimum which equal to zero of every functionals:

$$
\begin{aligned}
& S_{m}\left(x_{1}, ., x_{n}\right)=\sum_{v=1}^{n} \prod_{j=1}^{n} \prod_{l, p \in \Upsilon_{i, i=r_{v}}}((i+l+p \\
& \left.-x_{j-1}-x_{j}-x_{j+1}\right)^{2} \\
& +\left(m i+l+p-x_{j-1}-m x_{j}-x_{j+1}\right)^{2}+\Theta \\
& D_{m}\left(x_{1}, ., x_{n}\right)=\sum_{v=1}^{n} \prod_{j=1}^{n} \prod_{l, p \in \Upsilon_{i, i=r_{v}}}\left(\left(i / l p-x_{j} / x_{j-1} x_{j+1}\right)^{2}\right. \\
& \left.+\left(i^{2} / l p-x_{j}^{2} / x_{j+1} x_{j-1}\right)^{2}\right)+\Theta \\
& \text { where } \\
& \Theta=\left(R-\sum_{w+1}^{n} x_{w}\right)^{2}
\end{aligned}
$$

give to us natural numbers $x_{1}=t_{1}^{r}, . ., x_{n}=t_{n}^{r}$ for one of the $r$. Moreover, if there exist constant $\varepsilon \simeq 1$ and there value of $S_{m}$ or $D_{m}$ equal to $\varepsilon$ there are at list one Hamiltonian circuit.

Let's consider $D_{m}\left(x_{1}, . ., x_{n}\right)=0$ where every part of sum is equal to zero or on the other words for every unique number $i / l p$ exist equal value $x_{j} / x_{j-1} x_{j+1}$.
Hence, we can write $\alpha x_{j} / \beta x_{j-1} \gamma x_{j+1}=i / l p$. Then $\alpha=\beta \gamma$ but if we consider $\left(i / l p-x_{j} / x_{j-1} x_{j+1}\right)^{2}$ and $\left.\left(i^{2} / l p-x_{j}^{2} / x_{j+1} x_{j-1}\right)^{2}\right)$ we can write $\alpha=1$ and $x_{j}=i$ so every part of sum which equal to zero related with only one number $j$. Also, number of same parts is equal to $n$. We can write $l p=x_{j-1} x_{j+1}$ and factors of product are natural numbers related with other clauses then $x_{j-1}=l$ and $x_{j+1}=p$.

We can say $x_{j-1}, x_{j}, x_{j+1}$ are vortexes $l, i, p$ part of Hamiltonian Circuit. If it's not then there are three other natural numbers $x_{j_{1}-1}, x_{j_{1}}, x_{j_{1}+1}$ marked other part of the circle $x_{1}, x_{2}, \ldots, x_{n}$. Then $x_{j_{1}}$ is equal to $i$ but $x_{j_{1}-1}$ is not equal to $l$. Hence there are not enough numbers of the 'thirds' for every $i$.

For $S$ proof is same so sums are similar for products of $D$.

How we can solve problem numerically? We can consider stationary point conditions:

$$
\frac{\partial S}{\partial x_{j}}=0 j=1, . . n
$$

as the system of nonlinear equations where unknowns are $x_{j}$. It's more effective approach then consideration of $\nabla D$. We can solve it with help of some kinds of well known low relaxation methods.

Note, our problem is NP-complete and problem of global extremum NP-complete too. But if we find exact global minimum which equal to $\varepsilon$ we can tested problem for some large $m$ and it can give to as part of the answer for $c o-N P$ problem - Hamiltonian Graph.

Let us consider prime numbers $r_{i}$. Then we can write.

Theorem 0.2 Let us consider two graphs $G_{1} G_{2}$. Numbers of the vertexes $G_{1}$ are $r_{i}$. Numbers of the vertexes of $G_{2}$ are natural numbers $j=1,2,, . . n$ and for every $j$ related unknown weight $x_{j}$.

If there exist vector $x_{1}, . ., x_{n}$ where $I_{m}\left(x_{1}, . ., x_{n}\right)=0$ and function with $m \geq 2$

$$
I_{m}\left(x_{1}, . ., x_{n}\right)=\sum_{v=1}^{n} \prod_{j=1}^{n}\left((i) \prod_{p \in \Upsilon_{i, i=r_{v}}} p-x_{j} / \prod_{s \in \Upsilon_{j}} x_{s}\right)^{2}
$$




$$
\left.+\left(i^{m} / \prod_{p \in \Upsilon_{i, i=r_{v}}} p-x_{j}^{m} / \prod_{s \in \Upsilon_{j}} x_{s}\right)^{2}\right)
$$

then graphs $G_{k}$ are isomorphic and isomorphism can be described as $\phi: i \rightarrow j$, where $i=x_{j}$.

Modified form of $I_{m}$ :

$$
\begin{gathered}
\operatorname{SubI}_{m}\left(x_{1}, ., x_{n}\right)=\sum_{w=1, i=r_{w}}^{n} \prod_{j=1}^{n} \prod_{\left|\Omega_{i}\right|=\left|\Upsilon_{x_{j}}\right|}\left(\left(i / \prod_{l_{z} \in \Omega_{i}} l_{z}\right.\right. \\
\left.-x_{j} / \prod_{x_{v} \in \Upsilon_{x_{j}}} x_{v}\right)^{2}+\left(i^{m} / \prod_{l_{z} \in \Omega_{i}} l_{z}-x_{j}^{m} / \prod_{x_{v} \in \Upsilon_{x_{j}}} x_{v}\right)^{2} \\
\Omega_{i} \subseteq \Upsilon_{i}
\end{gathered}
$$

can give to us functional associated with SUBGRAPH ISOMORPHISM PROBLEM with same result.

\section{References}

[1] M. Blum, How to prove a Theorem So No One Else Can Claim It Proceedings of the International Congress of Mathematicians, Berkeley, CA, 1986, pp. 1444-1451.

[2] O. Coldreich, Proof that yield nothing but their validity or all languages in NP have zero-knowledge proof systems //J.ACM. V.38, No 3, 1991. P. 691729 . 